\title{
The Human Sweet Tooth
}

\section{Danielle R Reed* and Amanda H McDaniel}

\author{
Address: Monell Chemical Senses Center, Philadelphia PA 19104, USA \\ Email: Danielle R Reed* - reed@monell.org; Amanda H McDaniel - amcdaniel@monell.org \\ * Corresponding author
}

from Biotechnology and Biomaterials to Reduce the Caries Epidemic

Seattle, USA. 13-15 June 2005

Published: 10 July 2006

BMC Oral Health 2006, 6(Suppl I):SI7 doi:I0.II86/I472-683I-6-SI-SI7

(c) 2006 Reed and McDaniel; licensee BioMed Central Ltd.

This is an open access article distributed under the terms of the Creative Commons Attribution License (http://creativecommons.org/licenses/by/2.0), which permits unrestricted use, distribution, and reproduction in any medium, provided the original work is properly cited.

\begin{abstract}
Humans love the taste of sugar and the word "sweet" is used to describe not only this basic taste quality but also something that is desirable or pleasurable, e.g., la dolce vita. Although sugar or sweetened foods are generally among the most preferred choices, not everyone likes sugar, especially at high concentrations. The focus of my group's research is to understand why some people have a sweet tooth and others do not. We have used genetic and molecular techniques in humans, rats, mice, cats and primates to understand the origins of sweet taste perception. Our studies demonstrate that there are two sweet receptor genes (TASIR2 and TASIR3), and alleles of one of the two genes predict the avidity with which some mammals drink sweet solutions. We also find a relationship between sweet and bitter perception. Children who are genetically more sensitive to bitter compounds report that very sweet solutions are more pleasant and they prefer sweet carbonated beverages more than milk, relative to less bitter-sensitive peers. Overall, people differ in their ability to perceive the basic tastes, and particular constellations of genes and experience may drive some people, but not others, toward a caries-inducing sweet diet. Future studies will be designed to understand how a genetic preference for sweet food and drink might contribute to the development of dental caries.
\end{abstract}

\section{Introduction}

The sense of taste gives us important information about the nature and quality of food, and of all the basic taste qualities, sweetness is the most universally liked. The human appetite for refined sugar and for sweet foods and drinks has been so strong that it has influenced the course of human history, and the recent and sharp rise in the consumption of sugar may be unprecedented. However, although there is a strong desire on the part of humans to seek and ingest sweet foods and drinks, it would be inaccurate to view the liking and enjoyment of sweetness and sweeteners as uniform across people and populations. The reasons for these individual differences are largely unknown, but the explanation for these differences could have important public health consequences. Several variables are good predictors of how well sweets are liked, such as age (e.g., children are more avid consumers than are adults). The term "sweet tooth" has been coined to describe people who "prefer" sweets, implying that these individuals differ from those who "do not prefer" sweets. However, sweet perception varies even in the same individual over time, and therefore a well-conceived model of the sweet tooth must be able to account for this observation and a broad range of other variables (Figure 1). 


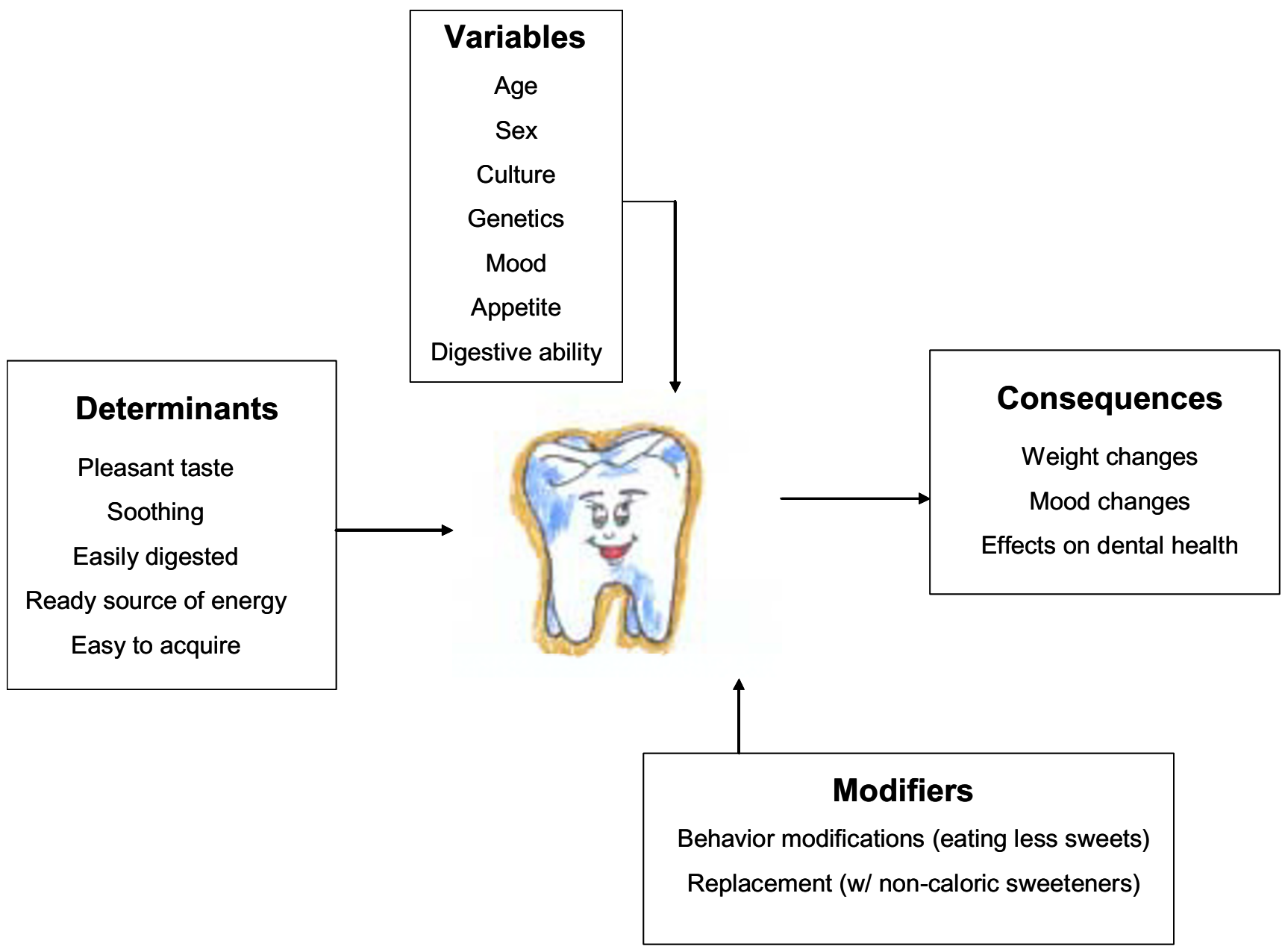

Figure I

The determinants and consequences of the sweet tooth. The aspects that determine how humans respond to sweets are listed in the box labeled "Determinants". Individual variables that may influence these determinants are listed in the box under the heading "Variables". The box labeled "Modifiers" denotes those methods used to modify or change our attitudes and behaviors toward sweets. In the box labeled "Consequences" we list the physical and psychological effects of eating sweets.

There are several facets of the human sweet tooth, and several academic disciplines, such as genetics, nutrition, and psychology, that contribute to our understanding of this trait. For instance, recent studies in mice demonstrate that DNA sequence variants in a sweet taste receptor gene have an effect on sweet preference and intake, and so it is possible that genetic differences among individuals may play a role in the human sweet tooth. Understanding sweetness also expands our understanding of bitterness. Bitter perception is related to sweet perception in humans, and these two sensory systems share some common features. The amount of calories we consume from sweet substances may play a role in the regulation of our body weight and overall nutritional status. Other variables help shape our behavior toward eating sweets and determine how many calories we consume from them, such as differ- ences in our ability to digest sugars. Additionally, the taste of sweet may be sought for its pleasurable and soothing properties, and thus may be consumed irrespective of the nutritional consequences. Our individual behaviors toward sweets and our underlying reasons for consuming sweets are complex; therefore, an extensive understanding of the sweet tooth will assist us in tailoring nutritional advice.

\section{The Role of the Sweet Tooth}

Some aspects of human experience are universally regarded as pleasant, and the experience of sweetness is a classic example. In many languages, the word for "sweet" connotes pleasant experience [1], and sugar has been introduced into many cultures where concentrated sweetness was unknown, with universal enthusiasm [2]. Our 
appetite for refined sugar and for sweet foods and drinks has been so strong that it has influenced the course of human history, and the recent and sharp rise in the consumption of sugar is perhaps unprecedented. This diet change has been the subject of popular books on the history and the culinary and biological effects of refined sugar [2-4].

An organizing explanation for the general attractiveness of sweets is that sugar has calories, and therefore sugar is a chemical signal for nutrients that can be readily detected and used for energy by animals. Furthermore, sugar also is a signal for safety because sweet items are rarely poisonous. Parenthetically, ethylene glycol, a chemical introduced into the environment by humans for use as antifreeze, is both sweet and poisonous and is responsible for a large proportion of deaths by accidental poisoning in dogs [5-7]. In nature, sweetness comes most often from honey and fruit and is processed from sugar cane and sugar beets. In some instances, sugar and sweetness are concentrated in the parts of plants that are meant to be eaten by animals so that the seeds can be dispersed, for example, dates or apples. Honey is an exception: it is an item that is meant to nourish bees but can be stolen by those willing to risk the consequences. Honey aside, highly concentrated sweet foods and drinks were not a part of the human diet until relatively recently. Today we refine sugar from sugar cane and sugar beets and also produce several artificial compounds to produce the sensation of sweet. The introduction of refined sugar into the lives of humans, first as a medicine and later as a food, has been full of consequences, not all of them desirable [8].

There is evidence to suggest that the liking for sweetness co-evolved with that for ethanol, because fermented fruit contains both [9]. Additional support for this hypothesis comes from the observation that ethanol and sweet perception share common neural substrates in rodents [10], and human alcoholics may have an increased sweet preference relative to those who are not alcoholics [11].

\section{Variation in Sweet Perception and Preference}

Sugar is consumed because it tastes pleasant and is a ready source of energy that can be used by the body. Nevertheless, there is more variation than is widely appreciated in both perception and preference [12]. Thus, to get a better understanding of the variation seen in our response to sweet, we need to determine the relative contribution of our ability to detect and how intensely we perceive the taste of sweeteners versus our actual preferences for them. Additionally, we must consider that sweet taste perception and preference can vary both between individuals and in the same individual over a period of time.

\section{Variation in detection and intensity}

Individuals differ in their ability to detect the taste of sweet at low concentrations $[13,14]$, and although rare, there are a few people who do not perceive a sweet taste from sucrose at all [15-17], a condition known as aglycogeusia. Likewise, the intensity ratings given for a single concentration of one sweet solution vary among people. The conscious perception of sweetness occurs when certain types of chemicals, such as sugars, contact taste receptor cells in the mouth. The taste receptor cell converts the chemical signal into an electrical one, and the nerve impulse is carried to the brain, where cortical taste areas are organized. Thus, the variation noted in the human ability to taste sweetness and the intensity with which it is perceived may be the result of differences in the taste receptor cells, in differences that occur along the pathway to the brain, or in the brain itself.

\section{Variation in preference and desire}

Aside from individual differences in our ability to detect and perceive the intensity of a sweet taste, there are large differences among people in the degree to which they like highly sweetened foods. Human subjects have been divided into two types. Type I responders are subjects who like increasing concentrations of sucrose up to a middle range of concentration, followed by a breakpoint after which preference decreases with increasing concentration. For these people, some foods and drinks are too sweet past a certain point, and this pattern is sometimes referred to as an inverted U-shape. The Type II response is characterized by increased liking as the concentration increases, which levels off but does not decrease as concentration increases further $[18,19]$. For these people, there is no such thing as too sweet. Although the classification of human subjects into two categories is probably too simple to capture the range of human responses to sweeteners, it highlights the dissimilarity among people.

\section{Differences among groups}

The perception and/or preference for sweet may vary not only from one individual to the next but also from one group to another. Studies have revealed effects of race, sex, and living situation (rural vs. urban). For instance, Americans of African descent prefer higher concentrations and Pima Indians prefer lower concentrations of sugar compared with those of European ancestry [20-25]. However, these racial differences may generalize only to specific food types. For instance, Taiwanese students rate sucrose solutions as more pleasant, but sweetened cookies as less pleasant, than do students of European descent [26]. Studies of sex differences suggest that male and female infants do not differ in sweet preference [20], but that older boys and men prefer higher concentrations of sweet compared with women $[27,28]$. Changes in the detection thresholds for sweet stimuli may tie into variations in sex 
hormone concentrations in women [29], but with conflicting effects on sucrose preference [30,31]. In addition to age and sex, the place someone lives may also influence sweet preference: Iranian children living in urban areas prefer much higher concentrations of sweetness in tea (a typical drink for these subjects) compared with children in rural areas [32].

\section{Change over time}

Variations in the perception of sweet may also occur within the same individual over time. These changes may be short term (e.g., changes in internal states) or long term (e.g., changes related to development and age).

Our need for sugar may dictate our perceptions and/or preferences for sweet, and day-to-day or periodic changes in our internal states may change this need. For example, the bodily fluctuations associated with our state of satiety that dictates our need for calories may influence our preference for sweet. When metabolic changes occur that reduce the availability of glucose in the blood, such as increases in plasma insulin concentration, then sweet preference increases [33-36]. Other longer-term changes to energy homeostasis in the body, such as weight gain or weight loss, may also alter our perception and/or preferences for sweet $(e . g .,[37,38]$. Leptin, a hormone secreted by adipocytes, is a signal to the brain to indicate high or low energy reserves [39], and its action both in the brain and on taste tissues may influence our need and preferences for energy-dense foods, including sweets (further discussed below under Biology and Genetics of Sweet Taste). Additionally, other types of internal states may alter our perception and/or preferences for sweets. Sweet has been purported to share neurochemical pathways with alcohol and other drugs stimulating endogenous opiates $[40,41]$; therefore, shifts in psychological states (e.g., periods of depression, emotional upset, or anxiety) may increase our sensitivity to and craving for the rewarding properties of sweet taste $[42,43]$.

Factors that produce more or less permanent modifications in our perception and/or preference for sweets may be the result of changes that occur as we develop and age. For instance, children are especially avid consumers of sweet foods and drinks. Babies are born liking sweet foods and drinks, as witnessed by facial expressions that display contentment and enjoyment $[44,45]$. Children prefer a higher concentration of sucrose in solution compared with their mothers, and this effect is true for at least two racial and ethnic groups [46], and perhaps applies to most or all cultures. However, the liking for concentrated sweetness fades during adolescence [21,47]. Although the reasons for this change are not known, they may have to do with changes in caloric requirements for growth, or the onset of puberty.
Overall, it is important to note that perception and preference are distinct measures. They may be related; for instance, in the simplest case, if someone could not perceive sweet taste (e.g., if granulated sugar tasted like sand), we would not expect that person to seek and consume sweet foods and drink. Thus, in studies measuring preference for sucrose at low concentrations, it is important to consider that some people will not be able to perceive the stimuli as well as others. However, perception and preference may also be unrelated except in the most extreme cases of perceptual loss. Thus far in human studies, sweet detection threshold does not predict either how intensely higher concentrations are perceived or how much they are liked [48-51]. In mice, there is a clear relationship between peripheral sensitivity and intake of sweeteners [52]; however, it appears that this relationship in humans is less clear.

\section{The Study and Measurement of the Sweet Tooth}

To gain a thorough understanding of both the differences and similarities among individuals in the perception of sweetness, and to form a more substantial model for determining its use as a nutrient, scientists have performed numerous studies and employed many different measures. The study of the human sweet tooth has been undertaken by psychophysicists who measure sensory thresholds and intensity ratings, psychologists who study the desirability and hedonic and addictive qualities of sweetness, molecular and cellular biologists who seek to understand the transduction machinery that turns a chemical signal (sugar in the mouth) to an electrical signal that is decoded in the brain, and nutritionists and physicians who study why people choose sweet foods as nutrients and the effects of this choice on health. The emphasis on sweet perception is motivated to no small extent by the almost drug-like desirability of sweetness, with the goal often being to find out how to give people an experience of sweetness without the negative consequences of too many calories and tooth decay.

\section{Methods to study sweet perception in humans}

Most tests of sweet perception use sugar dissolved in water as taste stimuli. However, tests can use foods or complex solutions $[53,54]$. The results of these tests are related to the type of food or drink tested, so, for instance, one person might prefer a lower concentration of sweetness in tomato soup and a higher concentration in a fruit-flavored drink, whereas another person might prefer the reverse. Whether one uses sugar dissolved in water or a more complex stimulus to measure sweet perception, laboratory stimuli may only partially generalize to other sweet foods and drinks and to behavior outside the laboratory [55-57]. 
Measurement of the sweet tooth can be categorized in the following way: (1) assessment of sensitivity, as in detection/recognition thresholds, perceived intensity, and quality description; and (2) hedonic assessment, as in pleasantness, preference and craving. Tests of taste sensitivity measure the ability to taste the stimulus and determine its quality, and tests of taste hedonics are designed to measure how pleasant the stimulus is and the desire to consume it. Sometimes both of these types of measures are used in a single study to gain a more comprehensive understanding of what factors contribute to the sweet tooth $[58,49]$. Also, sometimes DNA is collected and analyzed together with psychophysical and behavioral measures to uncover the genetics of sweet taste perception [57]. Additionally, researchers have used many of these tests in studies designed to understand what role the sweet tooth plays in nutritional status, and determine if it may be a contributing factor in disorders such as diabetes and obesity $[59,60]$.

Threshold tests measure the lowest concentration of a stimulus that a subject can detect or recognize [61]. In intensity tests, subjects are often asked to rate how intense a range of sweet concentrations is perceived to be, often relative to a standard. Quality tests require that the subject determine whether the stimulus is sweet, salty, sour, bitter, or savory. Sometimes these various measures are combined into one test. For instance, when measuring detection and recognition thresholds, the subject is asked to determine at what concentration he or she can detect the stimuli and then again at what concentration he or she is able to distinguish or "recognize" the taste as "sweet." Detection thresholds are almost always lower than recognition thresholds, because most subjects are able to determine if there is something in a solution before they can identify its quality.

There are several types of hedonic measures for taste stimuli. In one type of test, people individually rate a taste stimulus (e.g., sucrose) for pleasantness [62-66,19] or liking $[67,49,68]$. In other tests, subjects are offered two or more stimuli and asked to pick the one preferred [23]. Sometimes investigators ask subjects to rate a stimulus as being sweeter or less sweet than their ideal conception of what the stimulus should taste like [53] or ask them to rate themselves in terms of their level of sweet preference [69].

Learning how infants and children respond to different taste qualities both perceptually and affectively is a harder task because they simply do not have the language skills or the cognitive ability to relay their responses verbally or with paper-and-pencil tests. Therefore, to obtain data from young children, scientists rely on facial responses or other nonverbal responses. Facial responses are specific to certain taste qualities, and to a trained professional it is possible to decipher a facial expression that denotes pleasure versus pain, or one elicited from the taste of a sweet versus a bitter or sour stimuli [70-73] (reviewed in Erickson and Schulkin) [74]. These types of tests have also been used to look at the affective responses to tastes and smells in other animals, such as rats [75] and some types of primates $[73,76]$. The use of nonverbal measures of sensory perception or preference is not limited to children. Facial expressions are a useful addition to the study of adults [77]. Likewise, to get a more objective measure of physiological responses to taste stimuli, some investigators have studied heart rate changes or other responses not under voluntary control, such as sweating [71]. The use of these measures may partially circumvent the desire on the part of the subject to give a socially desirable response.

Understanding the sweet tooth also requires looking at the genes that are responsible for our ability to perceive the taste of sweet and the variations in these genes that contribute to individual differences in responses to sweet. Generally, scientists will ask subjects to perform psychophysical or behavioral tests and at the same time collect DNA. DNA can be collected from blood or from buccal cells that line the insides of the mouth, which is a good choice because it is less invasive than collecting blood [78]. DNA can then be genotyped to detect variants either in specific known genes (e.g., genes with a known involvement in sweet taste) or at a certain location along a chromosome that is predicted to harbor a gene involved in sweet taste. Once DNA is genotyped, researchers can ascertain whether responses on psychophysical or behavioral tests correlate with specific genotypes [57].

Nutritionists, physicians, and others interested in how the consumption of sweets influences health incorporate many of these perceptual and affective indices into their studies to help them understand why people choose to eat sweet foods and to determine if there are nutritional consequences to these choices [79]. There are various studies that examine the relationship between body weight and thresholds for sweet taste perception and/or preferences for sweets, reviewed in McDaniel and Reed, 2004 [80]. Some experiments test whether there is a correlation between body weight and the foods people eat, including sweets and fats, and also examine the concomitant effects of weight on thresholds and/or preferences for sweet stimuli. Others have people of various weights (e.g., underweight, normal weight, overweight, and obese) ingest a preload of a caloric substance and then have them rate their perception and/or preference for sweet stimuli. These tests help determine if there is in an interaction between immediate energy available as calories (preload) and long-term energy stores (body fat) that may influence sweet perception. Studies such as these help scientists and 
others understand the effects of body weight on the perception of sweet taste and, reciprocally, how sweet taste perception and preference may influence body weight.

To gain a more thorough understanding of how sweet perception and preferences interact with body weight, we need to establish how they translate into actual appetitive behaviors. Food diaries or questionnaires are useful in determining when, why, and where people choose to consume the foods that they eat $[81,82]$. They are also useful because they are a real-world measure, unlike one taken in the artificial setting of the laboratory. However, due to the disinclination of subjects to report all the foods they consume correctly [83], especially ones they may consider "naughty," such as highly sweetened candies and cookies, other proxy measures of sweet consumption can be used to evade reporter bias, such as the measurement of dental caries $[42,84]$.

These psychophysical and behavioral tests may be used with other indices, such as the measurement of appetite and weight-regulating hormones, to determine the physiology underlying the effect of body weight on the perception, preference, and appetite for sweet foods $[33,85]$. Combining these measures assists researchers in understanding the physiological mechanisms that are linked to our appetitive behaviors.

Although all of the above-mentioned measures have generated much knowledge on the sweet tooth, scientists must also be able to account for all variables that may influence how a subject responds on tests and the validity of these results in a real-world setting. As already noted, responses vary when taste tests are conducted on several different occasions $[86,87]$, although these changes in sweetness perception and preference are small compared with, for example, changes in olfactory threshold over time [88]. In addition to subject characteristics (e.g., ancestry, sex, and age), factors such as time of day, hunger, recent change in body weight, hormonal fluctuations, and testing conditions alter sweet perception and, therefore, need to be taken into account (for a review, see McDaniel and Reed, 2004)[80].

It is also important to remember that, although measures of sweet preference using sugar solutions are commonly used in the laboratory, they may not predict the preference for other sweeteners besides sugars [89] or selection of sweet foods or beverages at mealtime. Investigators have tried to bridge the gap between preference measures for laboratory stimuli and preference measures for foods and drinks by using mixtures of sugar and milk $[54,90]$ or by adding sugar to simple beverages or foods [53]. As observed from comparing self-reported eating behaviors, such as food diaries, with sweet preference measures inside the laboratory, there is not always complete agreement $[23,56]$.

Each type of test - threshold, perceived intensity, quality description, and hedonic - may measure both overlapping and unique perceptual processes that differ among individuals. Studies in which all of these measures are made in the same subjects are rare, and the extent to which individual differences in threshold, the ability to detect small increases in concentration, or perceived intensity affect pleasantness ratings has not been comprehensively studied. The studies to date have suggested these relationships are weak or non-existent $[49,91,92]$. However, the selective study of people at the extremes of sensitivity, high and low, may be needed to determine whether these relationships exist and how important they are for human sweet consumption.

\section{Biology and Genetics of Sweet Taste}

The initial events in the perception of sweet taste in humans occur in taste receptor cells on the tongue and palate, which are found clustered in taste buds in taste papillae. Taste papillae on which most sweet receptors reside can be seen on the tip of the tongue and look like raised pink bumps or nipples (from which they derive their Latin name), and the perception of sweet intensity is related to their density $[93,94]$. Inside the taste papillae, taste receptor cells produce proteins that participate in sweet taste transduction, and some of these proteins are inserted into the cell membrane to form taste receptors. Taste receptor cells within taste buds contain neuropeptides that may modify and modulate the activity of neighboring cells [95]. In mice, taste receptor cells synapse onto primary afferent fibers, and the signals are relayed to the gustatory cortex via the nucleus of the solitary tract, parabrachial nucleus, and thalamic gustatory area, with a concentration of sweet-activated neurons in the rostral fields of these regions [96].

In the human taste bud, some cells express sweet receptors and respond to sweetness, whereas other cells express bitter receptors and respond to bitter chemicals. If a taste receptor cell is changed so that one that normally would be sensitive to sweetness instead expresses a bitter receptor, the animal treats that particular bitter chemical as though it were sweet. This result indicates that the brain reads the input of certain cells on the tongue as sweet, regardless of how those cells are stimulated [97]. In fact, even in response to primary taste qualities, the pattern of individual brain activity is consistent when one person is measured on several occasions, but people vary from each other, with each person's pattern being nearly unique [98]. 
Inside the taste receptor cell, two proteins combine to create a sweet receptor $[99,100]$. The names of these proteins are T1R2 and T1R3, for taste receptor family 1, proteins 2 and 3, and the names of the associated genes for these proteins are TAS1R2 and TAS1R3 [101]. In humans, if T1R3 pairs with the first member of this family, T1R1, the receptor is sensitive to compounds such as monosodium glutamate that are described as savory or umami, a Japanese word that translates as "delicious." The gene coding for T1R3 was discovered with the aid of mapping experiments in mice. Inbred mouse strains differ in their intake of saccharin and other sweeteners, and the results of experiments in breeding suggested that an allele of a single gene was partially responsible for these differences [101]. Then, through positional cloning approaches, this gene was identified and found to be Tas1r3 [99,102-106].

Alleles of the mouse Tas $1 r 3$ gene are related to differences in the consumption of sweeteners [107]. Recordings of the peripheral taste nerves suggest that mice with the lowpreference Tas $1 r 3$ alleles exhibit lower nerve firing in response to saccharin or other sweeteners $[52,108]$. Removal of the Tas1r3 gene in mice results in a diminished response to most sweeteners [109], and when both genes, Tas1r2 and Tas1r3, are removed or "knocked out," the response to sweeteners disappears [110]. The results of mouse gene knockout studies disagree about the degree to which mice lacking these receptors learn to drink sweeteners if given periods of exposure to sweetened water. However, in mice unaccustomed to sweetened water, those with removal of one or both sweet receptors do not consume these solutions in preference to water. Likewise, domestic cats and tigers do not prefer sweet solutions compared with water. This lack of interest in sugar is probably because they cannot taste sweetness because the DNA sequence of one of the relevant receptor genes (TAS1R2) has decayed over time [111].

If DNA sequence variants have a large effect on the intake of sweeteners in mice and cats, then it is possible that this may also be true in humans. Because the peripheral neural responses of humans to sugars predict their verbal reports about sweet intensity [112], peripheral differences in taste sensitivity may be a component of human behavior toward sweetness. Cell-based assay systems have been developed to study the function of sweet receptors, and in experiments where the DNA sequence of the genes were modified, the results suggest that small changes in the specific amino acid sequence of in the human T1R2 and T1R3 protein can lead to differences in intracellular signaling in response to sweeteners $[113,114]$. These experiments, taken in conjunction with individual differences in the psychophysical responses to sweeteners [115-117], suggest that alleles of these receptors might influence sweet perception. A full range of human variation in sweet per- ception probably exists $[12,118,119]$, and genetic variation in taste receptor genes may account for some or many of these person-to-person differences.

How genetic differences in sweet perception or liking that might exist in humans might translate into food intake and food preference is unclear. A review of the literature concluded that, although there was evidence that the degree of liking or preference for individual food items, including sweet items such as candy or sugared coffee, was genetically determined, the pattern of macronutrient consumption, such as whether the person ate more carbohydrate than fat, was more heritable [120]. Since the publication of that review, two studies have reported that families demonstrated significant genetic linkage for sugar intake to several chromosomal locations $[121,122]$, one of which is near a sweet receptor gene (1p36). Overall, there is modest evidence that genetic differences among people might account for a portion of individual differences in the consumption of sweet foods and drinks.

There are explanations for person-to-person differences in taste function other than genetic differences. For instance, hormones modify taste receptor cell activity and differ in concentration among people. Leptin is one example: although its receptors are located primarily in the hypothalamus, several peripheral sites of action have been discovered. One such site is on the taste receptor cell itself, and studies in mice have demonstrated a role for leptin in suppressing the physiological and behavioral responses to sweets [123]. Such a response was noted in a study of the differences in preference for sweet solutions between wild-type (nonmutant) mice and the $d b / d b$ mouse, a genetic mutant lacking a functional copy of the gene that codes for the leptin receptor [124]. Mice were administered exogenous leptin, which, through its action on the sweet taste receptor, suppressed the neuronal activation to sweet stimuli in normal mice, which subsequently reduced their consumption of sweet solutions. However, neuronal activation was not suppressed in the $d b / d b$ mutant mice, and their consumption of sweets was not diminished [125].

As demonstrated in other studies that employ mice as model organisms, the extrapolation of mouse data to humans must be approached with caution. Despite this note of caution, further examination of whether differences in leptin concentration or activity might influence sweet perception and/or preference is warranted. Although one study found no relationship between plasma leptin concentration and sweet preference in obese women [85], the manipulation of plasma leptin concentrations in humans and their consequences for the perception and preferences for sweets may be a logical 
next step in unraveling how the body uses the perception of taste to regulate energy consumption.

Hormones and neurotransmitters that participate in higher-order cortical brain systems that organize the conscious perception of sweet or that regulate the rewarding properties of sweet may further serve to control our reaction to sweet chemicals (for a review, see Levine et al., 2003)[126]. Family studies of addiction have demonstrated that alcoholics and their family members prefer sweeter solutions than do nonalcoholic family members, suggesting that alcohol and sweeteners may share similar reward pathways in the brain [11]. The hypothesis that the rewarding properties of opioid-receptor-stimulating drugs and sweet taste are controlled by a common neural substrate is upheld further by the ability of naloxone, an opioid antagonist, to reduce both the positive properties of opiates and the preference for the taste of sweet $[127,128]$. Scientists are currently investigating alleles of genes that code for opioid receptors to determine if these alleles may be associated with the sensitivity to the rewarding properties of sugar and also to the suppressive abilities of naloxone.

\section{Bitter/Sweet Perception}

The perception of sweet has had an extensive role in our evolution, but no more so than the taste of bitter. Vegetables contain many toxic compounds and are often bitter as a consequence, although humans have been working to breed out the bitter components and enhance the sweet components of our foods since selective plant breeding and human agriculture began. Plants cannot run from predators and so have developed chemical defenses that work by causing illness when eaten: by reducing enzyme activity, irritating the mouth and gut, provoking an allergic response, or altering hormone metabolism [129]. Early humans learned to heat plants to the point that many bitter compounds were less bitter and less toxic, and to add sweeteners to bitter foods. Both practices have expanded the human food supply.

Sweetness is used to mask the bitterness of medicines, and one French aphorism summarizes despair as "an apothecary without sugar." Some aspects of bitter perception by humans are caused by genetic variation [130-133]. Children with a genetic sensitivity to some bitters report that they like more highly concentrated sweetness compared with children who are less genetically sensitive to bitter [57]. Therefore, individual differences in bitter sensitivity may influence sweet intake, especially in children.

\section{Our Behavior Toward Sweets and the Consequences}

The term "sweet tooth" has been coined to describe the preferences of a person who "likes" sweets, but it is less clear whether simply liking sweets can predict the ten- dency to consume more sweets. Our actual behavior toward choosing to consume sweet foods is harder to decipher and may stem both from our liking and desire for their taste and from the environment and culture in which we live. Furthermore, our behaviors toward sweets have consequences for our health, and these consequences provide feedback that could serve to either reinforce or alter our future behaviors toward their consumption.

As described earlier in this chapter, there are a variety of different responses to the taste of sweet, and these surely play a role in our motivation to eat sweets. However, another reason that sugar may be consumed is for benefits other than taste (e.g., digestible calories), and people might differ in their ability to utilize or benefit from calories in sugars (e.g., hereditary sugar intolerance) $[8,134]$. Because there are several types of naturally occurring sugars and enzymatic steps associated with the specifics of their digestion, people vary in their ability to digest sugars. A difficulty in digesting sugar may, consciously or unconsciously, change behavior toward sugar. Some people may even begin to find the taste of sweet unpalatable, because they begin to associate this taste with the negative digestive consequences caused by eating sweet foods [135]. This behavioral effect is called a conditioned taste aversion [136]. These behavioral changes may, in turn, have consequences on health. For example, people with hereditary fructose intolerance learn to avoid fructose; as a consequence, they often do not have dental caries or other secondary problems related to sugar consumption. About $10 \%$ of the native inhabitants of Greenland do not tolerate sucrose [134], but the consequences for diet and dental caries prevalence in this group are not known.

Behaviors toward sweets may also be formed by our psychological need for the rewarding properties of sugars. Sweets may alleviate depression and premenstrual symptoms and provide relief from the cravings for other drugs, because sweet taste releases opiates into the blood [126]. The pacification provided by sugar and sweets has been demonstrated to increase the liking of sweets [137] and may also translate into a behavioral tendency to consume more sweets. Studies that have examined the intake of sweets in women with psychiatric problems have reported that their consumption is increased compared with those without reported psychiatric problems [42]. Overall, sugar is consumed because of its pleasant taste, ease of digestion, and positive effect on mood, and these three reasons are not mutually exclusive; each probably makes a contribution to overall behavior.

People's behaviors toward sweets are also biased by circumstance and by their beliefs about the food or drink they taste. If people are told they are drinking a particular 
brand of soft drink, they will have a different pattern of brain activation compared with the activation seen when they drink the same drink but are not told the brand name, or if they are told the beverage is a different brand name [138]. Although the chemical stimulation from the beverage is the same, previous experience and the person's beliefs about a particular brand alter the response of the brain as the beverage is tasted. A humorous example of the power of learning and experience comes from a study of newly married women from Oaxaca, Mexico, who reported a change in their sweet preference after marriage that brought them closer to the preference of their mother-in-law [139]. Thus, learning from our families and culture whether sweets are either acceptable or unacceptable may influence our behavior toward liking and consuming them and may have consequences for our health.

Our willingness to consume sweet nutrients is also probably influenced by supposed or known dietary effects that we have encountered personally, been advised of by health professionals, or read in popular literature. The highly caloric nature of most nutrients that contain sugars has led many individuals to avoid or restrain their intake of such substances. In addition, adverse effects on dental health and the threat of other undesired health-related conditions may quell the desire to eat sweets. Thus, despite the almost universal liking for the "taste" of sweets, many find the idea of consuming them in excess to be "bittersweet." However, although a stigma toward excess sweet intake may exist, the actual negative effects of eating sweets are less clear and have been debated in the experimental literature.

One such perceived negative effect on health is the fear that excess sugar consumption leads to obesity. However, studies on the effects of sweet intake on body weight have yielded conflicting evidence, some demonstrating a negative correlation between sweet intake and body weight and some reporting the opposite, a positive correlation between sweet intake and body weight (for a review, see Hill and Prentice, 1995) [140]. Studies that have measured food intake outside of the laboratory have mostly reported that lean subjects consume more of their total calories from sugar than overweight or obese subjects. This is not surprising given that some heavier people may be restraining their intake of foods that they consider more "fattening." However, proxy indices of sugar intake, such as the number of dental caries lesions, conflict with this finding, indicating that overweight women actually eat more sweet foods than do lean women [42].

Research on the biochemistry of energy expenditure reveals the specific metabolic consequences of eating different food types, including sugars. In contrast to fats, sug- ars are biologically "expensive" to store and are therefore burned as calories before fats [141]. Thus, if we contrast a diet high in sugars to a diet high in fat, a sugar-rich diet would seem to indicate a higher rate of metabolism and produce a leaner person. However, our daily food intake is more complex, and many foods we consume that are high in sugar are also high in fat. In fact, according to one study, high sugar content improves the palatability of foods high in fat, which may cause one to consume more fatty food [54]. In addition, the rewarding pleasurable qualities of sweet foods may lead some to consume sweet foods in excess of dietary requirements, to self-soothe. Furthermore, the state of the food (i.e., liquid or solid) may play an additional role in appetitive behavior. In studies where subjects are asked to add sugar as a liquid supplement to their diets, they do not appear to compensate for the additional calories by decreasing their intake of other food sources [142]; however, when sugar is introduced into the diet as a solid, there is a subsequent decrease in the amount of additional calories that are consumed [143]. It appears that our choice to eat sweet foods either as a replacement or as an addition to other nutrients may be a more correct predictor of whether sweet foods will have an effect on body weight than are mere measurements of total sweet intake. Thus, both the attitudes we have toward eating sweets and our actual eating behaviors are important in our regulation of body weight.

Another well-known consequence of consuming excess sugars is the negative impact on dental health [144]. When sugar is consumed, particularly sucrose, naturally occurring bacteria inside the mouth interact with the sugar and produce acids that demineralize enamel on teeth. This demineralization process creates dental caries (lesions on teeth), which produce pain and, if left untreated, will erode and destroy teeth. The amount and severity of dental caries are positively correlated with sugar intake, and particularly with extrinsic sugars (e.g., sugars that are refined and added to foods). For example, foods such as bakery confections, including cookies, candies, and cakes, are much more cariogenic than are foods containing intrinsic sugars, such as milk and fruits. Some have concluded that sugar is no longer a large threat to dental health because of the addition of fluoride (a remineralizing agent) to drinking water and to toothpastes. However, studies have revealed that, even in those receiving treatments of fluoride, a strong positive correlation remains between high sugar consumption and the prevalence of caries. Nevertheless, the combination of fluoride and lowered sugar intake does appear to have a synergistic effect on the reduction of dental caries. Keeping this in mind, it is important to note that dental caries is a progressive chronic disease that, through preventative measures such as lowering sugar intake and brushing with 
fluoride, can be stopped or at least delayed for long enough to prevent severe complications.

\section{Modifying Our Sweet Tooth}

To combat some of the either real or perceived problems with a sugar-laden diet, researchers have been looking for ways to modify our sweet tooth to reduce sugar intake. One way to reduce our need and intake of sugar is by replacing it with high-intensity sweeteners. We have been adept at harnessing a diverse array of chemicals that have a sweet taste but that contain few calories [145]. Because the use of low-calorie, high-intensity sweeteners by humans has a short evolutionary history, the long-range consequences of uncoupling sweetness from calories and introducing new food additives are unclear. One concern is that oral sweetness, for example from aspartame-sweetened gum, prepares the body for calories that it does not then receive and thus may exacerbate hunger [146]. Another concern is that high-intensity sweeteners may cause cancer or other negative health effects.

An alternative route to reducing the negative effects of sugar intake has been to attempt to reduce or enhance sweet preference through experience early in life. Many people, parents particularly, try to reduce the sugar preference of their children by reducing their children's intake of sweets, especially in infants and very young children. However, animal studies that have tried to reduce sweet liking by changing earlier experiences with sugar have thus far been unsuccessful [147-149]. Whether children who eat little sugar in childhood will like sugar less as adults is not known.

\section{Conclusion}

The sweet tooth is culturally universal and has played an important role in human evolution. However, the perception of sweet differs greatly across individuals and groups, and behaviors and preferences toward sweet are affected by an entire range of variables, from genetics and age to personal experiences and cultural beliefs. Studies of sweet perception have increased our understanding of individual differences, and genetic studies are expanding our knowledge even more. Finding ways of combining different types of measures into single studies gives us a more comprehensive understanding of the sweet tooth and the role it plays in our lives, including the consequences on our health. We hope, with continued and focused study, to unravel more of the mysteries that surround our sweet tooth so that we may find more effective ways to shape and modify it for our well-being.

\section{Competing interests}

The author(s) declare that they have no competing interests.

\section{Authors' contributions}

All authors read and approved the final manuscript

\section{Acknowledgements}

Michael G Tordoff, Julie A Mennella, Beverly J Cowart and Gary K Beauchamp commented upon earlier drafts of this chapter. The editorial advice of Patricia J Watson is gratefully acknowledged.

\section{References}

I. Chamberlain AF: Primitive taste-words. Am J Psychol 1903, I4(34):410-417.

2. Mintz SW: Sweetness and Power. New York: Viking Penguin; 1985.

3. McGee $\mathrm{H}$ : On food and cooking: the science and lore of the kitchen. New York: Scribner; 2004.

4. Yudkin J: Sweet and dangerous. Natl Health Federation 1978

5. Mueller DH: Epidemiologic considerations of ethylene glycol intoxication in small animals. Vet Hum Toxicol I982, 24(1):2 I-24.

6. Rowland J: Incidence of ethylene glycol intoxication in dogs and cats seen at Colorado State University Veterinary Teaching Hospital. Vet Hum Toxicol I987, 29(I):41-44.

7. Thrall MA, Grauer GF, Mero KN: Clinicopathologic findings in dogs and cats with ethylene glycol intoxication. J Am Vet Med Assoc 1984, 184(I):37-4I.

8. Cox TM: The genetic consequences of our sweet tooth. Nat Rev Genet 2002, 3(6):48I-487.

9. Dudley R: Evolutionary origins of human alcoholism in primate frugivory. $Q$ Rev Biol 2000, 75(I):3-15.

10. Lemon CH, Brasser SM, Smith DV: Alcohol activates a sucroseresponsive gustatory neural pathway. J Neurophysiol 2004, 92(1):536-544.

II. Kampov-Polevoy AB, Garbutt JC, Janowsky DS: Association between preference for sweets and excessive alcohol intake: a review of animal and human studies. Alcohol Alcohol 1999, 34(3):386-395.

12. Bartoshuk LM: Sweetness: History, preference and genetic variability. Food Technol I99I, 45(I I): I08-II3.

13. Blakeslee AF, Salmon TN: Genetics of sensory thresholds: individual taste reactions for different substances. Proc Natl Acad Sci USA 1935, $21: 84-90$.

14. Kahn SG: Taste perception - individual reactions to different substances. 195I, 44:263-269.

15. Henkin RI, Shallenberger RS: Aglycogeusia: the inability to recognize sweetness and its possible molecular basis. Nature 1970, 227(26I):965-966.

16. Knowles D, Johnson PE: A study of the sensitiveness of prospective food judges to the primary tastes. Food Res 194I, 6:207-216.

17. Richter C: The self-selection of diets. Essays in Biology: University of California Press; 1943:50 I-505.

18. Pangborn RM: Individual variation in affective responses to taste stimuli. Psychon Sci 1970, 2 I(2): 125-126.

19. Thompson DA, Moskowitz HR, Campbell RG: Effects of body weight and food intake on pleasantness ratings for a sweet stimulus. J Appl Physiol 1976, 4I(1):77-83.

20. Beauchamp GK, Moran M: Dietary experience and sweet taste preference in human infants. Appetite 1982, 3(2): 139-152.

21. Desor JA, Greene LS, Maller O: Preferences for sweet and salty in 9- to 15 -year-old and adult humans. Science 1975, 190(42। 5):686-687.

22. Greene LS, Desor JA, Maller O: Heredity and experience: their relative importance in the development of taste preference in man. J Comp Physiol Psychol 1975, 89(3):279-284.

23. Liem DG, Mennella JA: Sweet and sour preferences during childhood: role of early experiences. Dev Psychobiol 2002, 4I(4):388-395.

24. Salbe AD, DelPArigi A, Pratley RE, Drewnowski A, Tataranni PA: Taste preferences and body weight changes in an obesityprone population. AJCN 2004, 79(3):372-378.

25. Schiffman SS, Graham BG, Sattely-Miller EA, Peterson-Dancy M: Elevated and sustained desire for sweet taste in African-Americans: a potential factor in the development of obesity. Nutrition 2000, 16(10):886-893.

26. Bertino M, Beauchamp GK, Jen K-IC: Rated taste perception in two cultural groups. Chem Senses 1983, 8:3-15. 
27. Parker S, Kamel N, Zellner D: Food craving patterns in Egypt: comparisons with North America and Spain. Appetite 2003 , 40(2): 193-195.

28. Zellner DA, Garriga-Trillo A, Rohm E, Centeno S, Parker S: Food liking and craving: A cross-cultural approach. Appetite 1999, 33(I):6I-70.

29. Than TT, Delay ER, Maier ME: Sucrose threshold variation during the menstrual cycle. Physiol Behav 1994, 56(2):237-239.

30. Dippel RL, Elias JW: Preferences for sweet in relationship to use of oral contraceptives and pregnancy. Horm Behav 1980 , | 4(I): I-6.

31. Weizenbaum F, Benson B, Solomon L, Brehony K: Relationship among reproductive variables, sucrose taste reactivity and feeding behavior in humans. Physiol Behav 1980 24(6): $1053-1056$

32. Jamel HA, Sheiham A, Cowell CR, Watt RG: Taste preference for sweetness in urban and rural populations in Iraq. I Dent Res 1996, 75(I I): 1879-1884.

33. Briese E, Quijada M: Sugar solutions taste better (positive alliesthesia) after insulin [proceedings]. J Physiol 1978, 285:20P-2IP.

34. Mayer-Gross W, Walker JW: Taste and selection of food in hypoglycemia. Br J Exp Pathol 1946, 27:297-305.

35. Rodin J, Wack J, Ferrannini E, DeFronzo RA: Effect of insulin and glucose on feeding behavior. Metabolism 1985, 34(9):826-83 I

36. Thompson DA, Campbell RG: Hunger in humans induced by 2 deoxy-D-glucose: glucoprivic control of taste preference and food intake. Science 1977, I98(432 I): 1065-1068.

37. Drewnowski A, Holden-Wiltse J: Taste responses and food preferences in obese women: effects of weight cycling. Int J Obes Relat Metab Disord 1992, I 6(9):639-648.

38. Schiffman SS, Musante G, Conger J: Application of multidimensional scaling to ratings of foods for obese and normal weight individuals. Physiol Behav 1978, 2 I(3):4I7-422.

39. Friedman JM, Halaas JL: Leptin and the regulation of body weight in mammals. Nature 1998, 395(6704):763-770.

40. Berridge KC: Pleasures of the brain. Brain Cogn 2003 , 52(I): $106-128$.

4I. Hajnal A, Smith GP, Norgren R: Oral sucrose stimulation increases accumbens dopamine in the rat. Am J Physiol Regul Integr Comp Physiol 2004, 286(I):R3 I-7

42. Barkeling B, Andersson I, Lindroos AK, Birkhed D, Rossner S: Intake of sweet foods and counts of cariogenic microorganisms in obese and normal-weight women. Eur J Clin Nutr 2001, 55( I 0):850-855

43. Striegel-Moore RH, Morrison JA, Schreiber G, Schumann BC, Crawford $\mathrm{PB}$, Obarzanek $\mathrm{E}$ : Emotion-induced eating and sucrose intake in children: the NHLBI Growth and Health Study. Int J Eat Disord 1999, 25(4):389-398.

44. Desor JA, Maller O, Turner RE: Taste in acceptance of sugars by human infants. J Comp Physiol and Psychol I 973, 84:496-50I.

45. Steiner JE: Human facial expressions in response to taste and smell stimulation. In Advances in Child Development and Behavio Edited by: Reese H, Lipsitt L. New York: Academic Press; 1979:257-295.

46. Pepino MY, Mennella JA: Factors contributing to individual differences in sucrose preference. Chem Senses 2005, 30(Suppl I(i3)): 19-20.

47. Desor JA, Beauchamp GK: Longitudinal changes in sweet preferences in humans. Physiol Behav 1987, 39(5):639-64I.

48. Adams D, Butterfield N: Taste thresholds and caries experience. J Dent |979, 7(3):208-2 II.

49. Lundgren B, Jonsson B, Pangborn RM, Sontag AM: Taste discrimination vs hedonic response to sucrose in coffee beverage. An interlaboratory study. Chem Senses 1978, 3(3):249-265.

50. Mattes RD: Gustation as a determinant of ingestion: methodological issues. Am J Clin Nutr 1985, 4 I (4):672-683.

5I. Nilsson B, Holm A-K, Sjostrom R: Taste thresholds, preferences for sweet taste and dental caries in 15-year-old children. A pilot study. Swed Dent J 1982, 6:21-27.

52. Bachmanov AA, Reed DR, Ninomiya $Y$, Inoue M, Tordoff MG, Price RA, Beauchamp GK: Sucrose consumption in mice: major influence of two genetic loci affecting peripheral sensory responses. Mamm Genome 1997, 8:545-548.

53. Conner MT, Haddon AV, Pickering ES, Booth DA: Sweet tooth demonstrated: individual differences in preference for both sweet foods and foods highly sweetened. I Appl Psychol I988, 73(2):275-280

54. Drewnowski A, Brunzell JD, Sande K, Iverius PH, Greenwood MR: Sweet tooth reconsidered: taste responsiveness in human obesity. Physiol Behav 1985, 35(4):617-622.

55. Holt S, Cobiac L, Beaumont-Smith N, Easton K, Best D: Dietary habits and the perception and liking of sweetness among Australian and Malaysian students: A cross-cultural study. Food Quality and Preference 2000, I I:299-3 I 2.

56. Mattes RD, Mela DJ: Relationships between and among selected measures of sweet-taste preference and dietary intake. Chem Senses 1986, I I (4):523-539.

57. Mennella JA, Pepino MY, Reed DR: Genetic and environmental determinants of bitter perception and sweet preferences. Pediatrics 2005, I I 5(2):e2 | 6-222.

58. Langwill KE: Taste perception and taste preferences of the consumer. Food technol 1949, 3(4): I36-139.

59. Grinker J, Hirsch J, Smith DV: Taste sensitivity and susceptibility to external influence in obese and normal weight subjects. J Pers Soc Psychol 1972, 22(3):320-325.

60. Tepper BJ, Hartfiel LM, Schneider SH: Sweet taste and diet in type II diabetes. Physiol Behav 1996, 60(I): |3-18

61. Moskowitz HR: The psychology of sweetness. In Sugars in Nutrition Edited by: Sipple H, McNutt K. New York: Academic Press; 1974.

62. Frijters JER, Rasmussen-Conrad EL: Sensory discrimination, intensity perception, and affective judgment of sucrosesweetness in the overweight. J Gen Psychol 1982, 107:233-247.

63. Kampov-Polevoy A, Garbutt JC, Janowsky D: Evidence of preference for a high-concentration sucrose solution in alcoholic men. Am J Psychiatry I997, I 54(2):269-270.

64. Kleifield El, Lowe MR: Weight loss and sweetness preferences: the effects of recent versus past weight loss. Physiol Behav 1991, 49(6): $1037-1042$

65. Malcolm R, O'Neil PM, Hirsch AA, Currey HS, Moskowitz G: Taste hedonics and thresholds in obesity. Int J Obes 1980, 4(3):203-2I 2

66. Rodin J, Moskowitz HR, Bray GA: Relationship between obesity, weight loss, and taste responsiveness. Physiol Behav 1976, I 7(4):59|-597.

67. Looy H, Callaghan S, Weingarten HP: Hedonic response of sucrose likers and dislikers to other gustatory stimuli. Physiol Behav 1992, 52(2):219-225.

68. Pfaffmann C: The sensory and motivating properties of the sense of taste. In Nebraska Symposium on Motivation Edited by: Jones MR. University of Nebraska Press; 1961.

69. Partanen J, Bruun K, Markkanen T: Inheritance of drinking behavior. Helsinki: Keskuskirjapaino; 1966.

70. de Araujo IE, Rolls ET, Kringelbach ML, McGlone F, Phillips N: Tasteolfactory convergence, and the representation of the pleasantness of flavor, in the human brain. Eur J Neurosci 2003 I 8(7):2059-2068.

7I. Robin O, Rousmans S, Dittmar A, Vernet-Maury E: Gender influence on emotional responses to primary tastes. Physiol Behav 2003, 78(3):385-393.

72. Rosenstein $D$, Oster $H$ : Differential facial responses to four basic tastes in newborns. Child Dev 1988, 59(6): I555-1568.

73. Steiner JE, Glaser D, Hawilo ME, Berridge KC: Comparative expression of hedonic impact: affective reactions to taste by human infants and other primates. Neurosci Biobehav Rev 2001, 25(I):53-74.

74. Erickson K, Schulkin J: Facial expressions of emotion: a cognitive neuroscience perspective. Brain Cogn 2003, 52(I):52-60.

75. Grill HJ, Norgren R: The taste reactivity test. I.Mimetic responses to gustatory stimuli in neurologically normal rats. Brain Res 1978, I43(2):263-279.

76. Ueno A, Ueno Y, Tomonaga M: Facial responses to four basic tastes in newborn rhesus macaques (Macaca mulatta) and chimpanzees (Pan troglodytes). Behav Brain Res 2004, I 54(I):26I-27I.

77. Looy $\mathrm{H}$, Weingarten HP: Facial expressions and genetic sensitivity to 6-n-propylthiouracil predict hedonic response to sweet. Physiol Behav 1992, 52(I):75-82.

78. Saftlas AF, Waldschmidt M, Logsden-Sackett N, Triche E, Field E. Optimizing buccal cell DNA yields in mothers and infants for human leukocyte antigen genotyping. Am J Epidemiol 2004 I 60(I):77-84 
79. Hetherington MM: The physiological-psychological dichotomy in the study of food intake. Proc Nutr Soc 2002, 61 (4):497-507.

80. McDaniel AH, Reed DR: The human sweet tooth and its relationship to obesity. In Genomics and Proteomics in Nutrition Edited by: Moustaid-Moussa N, Berdanier C. New York: Marcel Dekker, Inc; 2004:49-67.

81. de Castro JM: Genetic influences on daily intake and meal patterns of humans. Physiol Behav 1993, 53(4):777-782.

82. Geiselman PJ, Anderson AM, Dowdy ML, West DB, Redmann SM, Smith SR: Reliability and validity of a macronutrient self-selection paradigm and a food preference questionnaire. Physiol Behav 1998, 63(5):919-928.

83. Lindroos AK, Lissner L, Sjostrom L: Validity and reproducibility of a self-administered dietary questionnaire in obese and non-obese subjects. Eur / Clin Nutr 1993, 47:46I-48I.

84. Barkeling $B$, Linne $Y$, Lindroos AK, Birkhed D, Rooth P, Rossner S: Intake of sweet foods and counts of cariogenic microorganisms in relation to body mass index and psychometric variables in women. Int J Obes Relat Metab Disord 2002, 26(9): 1239-1244.

85. Karhunen L, Lappalainen R, Haffner S, Valve R, Tuorila H, Miettinen $H$, Uusitupa M: Serum leptin, food intake and preferences for sugar and fat in obese women. Int J Obes Relat Metab Disord I998, 22:8|9-82|.

86. Stevens JC, Cruz LA, Hoffman JM, Patterson MQ: Taste sensitivity and aging: high incidence of decline revealed by repeated threshold measures. Chem Senses 1995, 20(4):45I-459.

87. Williams RJ: Biochemical individuality. New Canaan: Keats Publishing, Inc; 1956.

88. Dalton P, Doolittle N, Breslin PA: Gender-specific induction of enhanced sensitivity to odors. Nat Neurosci 2002, 5(3):199-200.

89. Moskowitz HR, Dubose C: Taste intensity, pleasantness, quality of aspartame, sugars and their mixtures. J Inst Can Sci Technol Aliment 1977, I0(2):|26-|3|.

90. Drewnowski A: Sensory preferences and fat consumption in obesity and eating disorders. In: Dietary Fats: Determinants of Preference, Selection, and Consumption. Edited by: Mela Essex: Elsevier Science; 1992:59-77.

91. Kocher EC, Fisher GL: Subjective intensity and taste preference. Percept Mot Skills 1969, 28(3):735-740.

92. Moskowitz HR, Kluter RA, Westerling J, Jacobs HL: Sugar sweetness and pleasantness: evidence for different psychological laws. Science 1974, I 84( I 36):583-585

93. Miller IJ Jr, Reedy FE Jr: Variations in human taste bud density and taste intensity perception. Physiol Behav 1990 47(6): $1213-1219$

94. Stein N, Laing DG, Hutchinson I: Topographical differences in sweetness sensitivity in the peripheral gustatory system of adults and children. Brain Res Dev Brain Res 1994, 82(I2):286-292.

95. Zhao FL, Shen T, Kaya N, Lu SG, Cao Y, Herness S: Expression, physiological action, and coexpression patterns of neuropeptide $\mathbf{Y}$ in rat taste-bud cells. Proc Natl Acad Sci USA 2005 , I02(3 I): I I I00-III 05.

96. Sugita M, Shiba Y: Genetic tracing shows segregation of taste neuronal circuitries for bitter and sweet. Science 2005 , 309(5735):78I-785

97. Mueller KL, Hoon MA, Erlenbach I, Chandrashekar J, Zuker CS, Ryba $\mathrm{N}$ ): The receptors and coding logic for bitter taste. Nature 2005, 434(7030):225-229.

98. Schoenfeld MA, Neuer G, Tempelmann C, Schussler K, Noesselt T, Hopf JM, Heinze HJ: Functional magnetic resonance tomography correlates of taste perception in the human primary taste cortex. Neuroscience 2004, I 27(2):347-353.

99. Li X, Staszewski L, Xu H, Durick K, Zoller M, Adler E: Human receptors for sweet and umami taste. Proc Natl Acad Sci 2002 99:4692-4696

100. Nelson G, Hoon MA, Chandrashekar J, Zhang Y, Ryba NJ, Zuker CS Mammalian sweet taste receptors. Cell 200I, I 06(3):38I-390.

101. Liao J, Schultz PG: Three sweet receptor genes are clustered in human chromosome I. Mamm Genome 2003, I 4(5):29I-30I.

102. Fuller JL: Single-locus control of saccharin preference in mice. Hered 1974, 65:33-36.

103. Bachmanov AA, Li X, Reed DR, Ohmen JD, Li S, Chen Z, Tordoff MG de Jong PJ, Wu C, West DB, Chatterjee A, Ross DA, Beauchamp GK:
Positional cloning of the mouse saccharin preference (Sac) locus. Chem Senses 200I, 26(7):925-933.

104. Kitagawa M, Kusakabe Y, Miura H, Ninomiya Y, Hino A: Molecular genetic identification of a candidate receptor gene for sweet taste. Biochem Biophys Res Commun 200I, 283(I):236-242.

105. Reed DR, Li S, Li X, Huang L, Tordoff MG, Starling-Roney R, Taniguchi K, West DB, Ohmen JD, Beauchamp GK, Bachmanov AA: Polymorphisms in the taste receptor gene (Tas Ir3) region are associated with saccharin preference in $\mathbf{3 0}$ mouse strains. Neurosci 2004, 24(4):938-946.

106. Max M, Shanker YG, Huang L, Rong M, Liu Z, Campagne F, Weinstein $\mathrm{H}$, Damak S, Margolskee RF: Tas Ir3, encoding a new candidate taste receptor, is allelic to the sweet responsiveness locus Sac. Nat Genet 200I, 28(I):58-63.

107. Montmayeur JP, Liberles SD, Matsunami H, Buck LB: A candidate taste receptor gene near a sweet taste locus. Nat Neurosci 200I, 4(5):492-498.

108. Sainz E, Korley JN, Battey JF, Sullivan SL: Identification of a novel member of the TIR family of putative taste receptors. J Neurochem 200I, 77(3):896-903.

109. Inoue M, Reed DR, Li X, Tordoff MG, Beauchamp GK, Bachmanov AA: Allelic variation of the Tas Ir3 taste receptor gene selectively affects behavioral and neural taste responses to sweeteners in the F2 hybrids between C57BL/6ByJ and I29P3/J mice. J Neurosci 2004, 24(9):2296-2303.

110. Damak S, Rong M, Yasumatsu K, Kokrashvili Z, Varadarajan V, Zou S, Jiang P, Ninomiya Y, Margolskee RF: Detection of sweet and umami taste in the absence of taste receptor TIr3. Science 2003, 30I(5634):850-853

III. Zhao G, Zhang Y, Hoon MA, Chandrashekar J, Erlenbach I, Ryba NJ, Zuker CS: The receptors for mammalian sweet and umami taste. Cell 2003, I I5:255-266.

112. Li X, Li W, Wang H, Cao J, Maehashi K, Huang L, Bachmanov AA, Reed DR, Legrand-Defretin V, Beauchamp GK, Brand JG: Pseudogenization of a sweet-receptor gene accounts for cats' Indifference toward sugar. PLoS Genet 2005, I(I):e3.

113. Diamant H, Oakley B, Strom L, Wells C, Zotterman Y: A comparison of neural and psycohphysical responses to taste stimuli in man. Acta Physiol Scand 1965, 64:67-74.

II4. Jiang P, Ji Q, Liu Z, Snyder LA, Benard LM, Margolskee RF, Max M: The cysteine-rich region of TIR3 determines responses to intensely sweet protein. J Biol Chem 2004, 279(43):45068-45075.

II5. Schiffman SS, Cahn H, Lindley MG: Multiple receptor sites mediate sweetness: evidence from cross adaptation. Pharmacol Biochem Behav 198I, 15(3):377-388

I 16. Xu H, Staszewski L, Tang H, Adler E, Zoller M, Li X: Different functional roles of TIR subunits in the heteromeric taste receptors. Proc Natl Acad Sci USA 2004, I 1 (39): | 4258- 14263

I I7. Faurion A, Saito S, Mac Leod P: Sweet taste involves several distinct receptor mechanisms. Chem Senses 1980, 5:107-121.

1 18. Lawless HT, Stevens DA: Cross adaptation of sucrose and intensive sweeteners. Chem Senses 1983, 7(3/4):309-315.

119. Faurion A: Physiology of the sweet taste. In Progress in Sensory Physiology Edited by: Autrum H, Ottoson D, Perl E, Schmidt R, Shimazu $H$, Willis W. Berlin: Springer-Verlag; 1987.

120. Okoro EO, Brisibe F, Jolayemi ET, Hadizath Taimagari G: Taste sensitivity to sodium chloride and sucrose in a group of adolescent children in Northern Nigeria. Ethn Dis 2000, 10 (I):53-59.

121. Reed DR, Bachmanov AA, Beauchamp GK, Tordoff MG, Price RA: Heritable variation in food preferences and their contribution to obesity. Behav Genet 1997, 27(4):373-387.

122. Cai G, Cole SA, Bastarrachea-Sosa RA, Maccluer JW, Blangero J, Comuzzie AG: Quantitative trait locus determining dietary macronutrient intakes is located on human chromosome 2p22. Am J Clin Nutr 2004, 80(5): I4I0-14I4.

123. Collaku A, Rankinen T, Rice T, Leon AS, Rao DC, Skinner JS, Wilmore $\mathrm{JH}$, Bouchard C: A genome-wide linkage scan for dietary energy and nutrient intakes: the health, risk factors, exercise training, and genetics (HERITAGE) family study. $\mathrm{Am} / \mathrm{Clin}$ Nutr 2004, 79(5):88I-886.

124. Kawai K, Sugimoto K, Nakashima K, Miura H, Ninomiya Y: Leptin as a modulator of sweet taste sensitivities in mice. Proc Nat/ Acad Sci USA 2000, 97(20): I 1044- I 1049.

125. Ninomiya Y, Sako N, Imai Y: Enhanced gustatory neural responses to sugars in the diabetic $\mathrm{db} / \mathrm{db}$ mouse. Am J Physiol 1995, 269(4 Pt 2):R930-7. 
126. Shigemura N, Ohta R, Kusakabe $Y$, Miura H, Hino A, Koyano K, Nakashima K, Ninomiya $Y$ : Leptin modulates behavioral responses to sweet substances by influencing peripheral taste structures. Endocrinology 2004, I45(2):839-847.

127. Levine AS, Kotz CM, Gosnell BA: Sugars: hedonic aspects, neuroregulation, and energy balance. Am J Clin Nutr 2003, 78(4):834-42S.

128. Arbisi PA, Billington CJ, Levine AS: The effect of naltrexone on taste detection and recognition threshold. Appetite 1999, 32(2):24I-249.

129. Fantino M, Hosotte J, Apfelbaum M: An opioid antagonist, naltrexone, reduces preference for sucrose in humans. Am J Physiol I986, 25 I(I Pt 2):R9I-6.

130. Leopold AC, Ardrey R: Toxic substances in plants and the food habits of early man. Science 1972, I76(34):5I2-5I4.

131. Bufe B, Breslin PA, Kuhn C, Reed DR, Tharp CD, Slack JP, Kim UK, Drayna $D$, Meyerhof $W$ : The molecular basis of individual differences in phenylthiocarbamide and propylthiouracil bitterness perception. Curr Biol 2005, I 5(4):322-327.

132. Fox $A$ : The relationship between chemical composition and taste. Science I93I, 74:607.

133. Kim U, Jorgenson E, Coon H, Leppert M, Risch N, Drayna D: Positional cloning of the human quantitative trait locus underlying taste sensitivity to phenylthiocarbamide. Science 2003, 299: 122I-I 225.

134. Snyder LH: Inherited taste deficiency. Science |93I, 74:|5|-|52.

135. McNair A, Gudmand-Hoyer E, Jarnum S, Orrild L: Sucrose malabsorption in Greenland. $\mathrm{Br}$ Med J I972, 2(5804): |9-2I.

136. Davidenkov S: Inherited inability to eat sugar. J Heredity 1940, 3 I (5):5-7.

137. Garcia J, McGowan BK, Ervin FR, Koelling RA: Cues: their relative effectiveness as a function of the reinforcer. Science 1968, I 60(829):794-795.

138. Blass E, Fitzgerald E, Kehoe P: Interactions between sucrose, pain and isolation distress. Pharmacol Biochem Behav 1987, 26(3):483-489.

139. McClure SM, Li J, Tomlin D, Cypert KS, Montague LM, Montague PR: Neural correlates of behavioral preference for culturally familiar drinks. Neuron 2004, 44(2):379-387.

140. Messer E: Some like it sweet: estimating sweetness preferences and sucrose intakes from ethnographic and experimental data. American Anthropologist 1986, 88(3):637-647.

I4I. Hill JO, Prentice AM: Sugar and body weight regulation. Am J Clin Nutr 1995, 62(I Suppl):264S-273S. discussion 273S-274S

142. Flatt J: The biochemistry of energy expenditure. In Recent advances in obesity research: II. Proceedings of the 2nd International Congress on Obesity Edited by: Bray GA. Westport, CT: Technomic Publishing Company; 1978:21 I-227.

143. Tordoff MG, Alleva AM: Effect of drinking soda sweetened with aspartame or high-fructose corn syrup on food intake and body weight. Am J Clin Nutr 1990, 5 I (6):963-969.

144. DiMeglio DP, Mattes RD: Liquid versus solid carbohydrate: effects on food intake and body weight. Int J Obes Relat Metab Disord 2000, 24(6):794-800.

145. Sheiham A: Dietary effects on dental diseases. Public Health Nutr 200I, 4(2B):569-59I.

146. Schiffman SS, Gatlin CA: Sweeteners: state of knowledge review. Neurosci Biobehav Rev 1993, I 7(3):3 3 3-345

147. Tordoff MG, Alleva AM: Oral stimulation with aspartame increases hunger. Physiol Behav 1990, 47(3):555-559.

148. Bernstein IL, Fenner DP, Diaz J: Influence of taste stimulation during the suckling period on adult taste preference in rats. Physiol Behav 1986, 36(5):913-919.

149. Bertino $M$, Wehmer $F$ : Dietary influences on the development of sucrose acceptability in rats. Dev Psychobiol 198I, I 4(I): 19-28.

150. Wurtman JJ, Wurtman RJ: Sucrose consumption early in life fails to modify the appetite of adult rats for sweet foods. Science 1979, 205(4403):32।-322.

\begin{tabular}{|} 
Publish with BioMed Central and every \\
scientist can read your work free of charge \\
"BioMed Central will be the most significant development for \\
disseminating the results of biomedical research in our lifetime. " \\
Sir Paul Nurse, Cancer Research UK \\
Your research papers will be: \\
• available free of charge to the entire biomedical community \\
• peer reviewed and published immediately upon acceptance \\
• cited in PubMed and archived on PubMed Central \\
• yours - you keep the copyright \\
Submit your manuscript here: \\
http://www.biomedcentral.com/info/publishing_advasp
\end{tabular}

